\title{
Quantum Histories and Their Implications
}

\author{
ADRIAN KENT \\ Department of Applied Mathematics and Theoretical Physics, \\ University of Cambridge, \\ Silver Street, Cambridge CB3 9EW, U.K.
}

\begin{abstract}
Classical mechanics and standard Copenhagen quantum mechanics respect subspace implications. For example, if a particle is confined in a particular region $R$ of space, then in these theories we can deduce that it is confined in regions containing $R$. However, subspace implications are generally violated by versions of quantum theory that assign probabilities to histories, such as the consistent histories approach.

I define here a new criterion, ordered consistency, which refines the criterion of consistency and has the property that inferences made by ordered consistent sets do not violate subspace relations. This raises the question: do the operators defining our observations form an ordered consistent history? If so, ordered consistency defines a version of quantum theory with greater predictive power than the consistent histories formalism. If not, and our observations are defined by a non-ordered consistent quantum history, then subspace implications are not generally valid.
\end{abstract}

PACS: 03.65.Bz

1 Email: a.p.a.kent@damtp.cam.ac.uk 


\section{Introduction}

We take it for granted that we can infer quantitatively less precise statements from our observations. For example, if we know that an atom is confined in some region $R$ of space, we believe we are free to assume for calculational purposes only that it lies in some larger region containing $R$. Our understanding of the world and our interpretation of everyday experience tacitly rely on subspace implications of this general type: if a physical quantity is known to lie within a range $R_{1}$, then it lies in all ranges $R_{2} \supset R_{1}$.

In classical mechanics, subspace inferences follow from the correspondence between physical states and points in phase space: if the state of a system lies in a subset $S_{1}$ of phase space, it lies in all subsets $S_{2} \supset S_{1}$. Similarly, in Copenhagen quantum theory, they hold since if the state of a quantum system lies in a subspace $H_{1}$ of Hilbert space, it lies in all subspaces $H_{2} \supset H_{1}$.

However, neither classical mechanics nor (presumably) Copenhagen quantum theory is fundamentally correct. If the basic principles of quantum theory apply to the universe as a whole, then a post-Copenhagen interpretation of quantum theory seems to be needed, and any justification of the subspace implications must ultimately be given in terms of that interpretation. Though it may seem hard to imagine how to make sense of nature without allowing subspace inferences, there are versions of quantum theory in which they do not hold. In particular, this is true of recent attempts to develop history-based formulations of quantum theory, [1, 2, 3, 4, [5 which rely on the notion of consistent or decoherent sets of histories.

This paper suggests a way in which quantum theory can plausibly be interpreted 
via statements about histories, without violating subspace implications — the motivation being that both history-based interpretations and subspace implications seem natural. The interpretation which results, a refinement of the consistent histories approach based on ordered consistent sets of histories, is certainly not the ultimate answer to the problems of quantum theory. It does not solve the measurement problem, for example. But perhaps it is a step in the right direction, or at least in an interesting direction. It also helps to make precise the question as to what it would mean if subspace implications actually were violated in nature, which the last part of the paper examines.

The language of quantum histories may not necessarily be the right way to interpret the quantum theory of closed systems. Bohm theory [6] and dynamical collapse theories[7] are particularly interesting alternatives, for example. Quantum histories still play a rôle in these theories as usually interpreted, but it is certainly possible to interpret them so as to ascribe no ontological status to the evolving quantum state, in which case quantum histories have no ontological status either, and the orderings amongst them become physically irrelevant.

In this paper, which focusses on the relation between quantum histories and orderings, I take it for granted — as an interesting hypothesis, not a dogma — that quantum histories have some ontological status. I will focus on the consistent histories approach. This is not to suggest that there are no other interesting quantum histories approaches. Other ideas are outlined in Ref. [8], Section VII of Ref. [9], and Ref. [10], for example. But the discussion of this paper is best carried out within some specific formulation quantum theory, and consistent histories is the most widely studied example. As an approach to closed system 
quantum histories, it has many interesting features. Its main drawback is that it does not solve the measurement problem, which in the language of consistent histories takes the form of a set selection problem. The search for ways to refine the definition of consistency in order to solve, or at least reduce, the set selection problem is an independent motivation for the definition of ordered consistency proposed below.

\section{Partial Ordering of Quantum Histories}

We consider versions of quantum theory that assign probabilities to histories - i.e., to collections of events. There are several reasonably standard ways of representing an event in quantum theory. The simplest is as a projection operator, labelled by a particular time, corresponding to a statement about an observable in non-relativistic quantum mechanics. Thus, the statement that a particle was in the interval $I$ at time $t$ is represented by the projection

$$
P_{I}=\int_{x \in I}|x\rangle\langle x| d^{3} x .
$$

The representation of events as projections can be generalised to quantum effects [11, 12, 10], defined by operators $A$ such that $A$ and $I-A$ are both positive.2 Events can also be defined, at least formally, in the path integral version of quantum theory by dividing the set of paths into exclusive subsets. For example, by considering the appropriate integrals one can attach probabilities to the events that a particle did, or did not, enter a particular region of space-time 13]. Further generalisations have been discussed by Isham, Linden and collaborators. [5, 14, 15]

2 Different definitions can be found: this, the simplest, is adequate for our purposes. 
Each of these representations has a natural partial ordering. For projections, we take $A \geq B$ if the range of $B$ is a subspace of that of $A$. This corresponds to the logical implication of Copenhagen quantum theory already mentioned: if the state of a quantum system lies in the range of $B$, then it necessarily lies in the range of $A$. For quantum effects, we take $A \geq B$ if and only if $(A-B)$ is positive. For events defined by the position space path integral, we take the statement that a particle entered $R_{1}$ to imply the statement that it entered $R_{2}$ if $R_{1} \subseteq R_{2}$.

We define a quantum history as a collection of quantum events and extend the partial ordering to histories in the natural way. For example, if we take quantum histories in non-relativistic quantum mechanics to be defined by sequences of projections at different fixed times, we compare two quantum histories $H=\left\{A_{1}, t_{1} ; \ldots ; A_{n} ; t_{n}\right\}$ and $H^{\prime}=\left\{A_{1}^{\prime}, t_{1}^{\prime} ; \ldots ; A_{n^{\prime}}^{\prime}, t_{n^{\prime}}^{\prime}\right\}$ as follows. First add to each history the identity operator at every time at which it contains no proposition and the other does, so obtaining relabelled representations of the histories as $H=\left\{A_{1}, T_{1} ; \ldots ; A_{N} ; T_{N}\right\}$ and $H^{\prime}=\left\{A_{1}^{\prime}, T_{1} ; \ldots ; A_{N}^{\prime}, T_{N}\right\}$. Then define the partial ordering by taking $H \geq H^{\prime}$ if and only if $A_{i} \geq A_{i}^{\prime}$ for all $i$ and $H>H^{\prime}$ if $H \geq H^{\prime}$ and $H \neq H^{\prime}$. This ordering was first considered in the consistent histories literature by Isham and Linden[14], whose work, and its relation to the ideas of this paper, are discussed in the Appendix.

\section{Consistent Histories}

The consistent sets of histories for a closed quantum system are defined in terms of the space of states $\mathcal{H}$, the initial density matrix $\rho$, and the hamiltonian $H$. In the simplest version of the consistent histories formulation of non-relativistic quantum mechanics, sets 
of histories correspond to sets of projective decompositions. In order to be able to give a physical interpretation of any of the consistent sets, we need also to assume that standard observables, such as position, momentum and spin, are given. Individual quantum events are defined by members of projective decompositions of the identity into orthogonal hermitian projections $\sigma=\left\{P^{i}\right\}$, with

$$
\sum_{i} P^{i}=1 \quad \text { and } \quad P^{i} P^{j}=\delta_{i j} P^{i} .
$$

Each such decomposition defines a complete and exclusive list of events at some fixed time, and a time label is thus generally attached to the decompositions and the projections: the labels are omitted here, since the properties of interest here depend only on the time ordering of events.

Suppose now we have a set of decompositions $\mathcal{S}=\left\{\sigma_{1}, \ldots, \sigma_{n}\right\}$. Then the histories given by choosing one projection from each of the decompositions $\sigma_{j}$ in all possible ways define an exhaustive and exclusive set of alternatives. We follow Gell-Mann and Hartle's definitions, and say that $\mathcal{S}$ is a consistent (or medium decoherent) set of histories if

$$
\operatorname{Tr}\left(P_{n}^{i_{n}} \ldots P_{1}^{i_{1}} \rho P_{1}^{j_{1}} \ldots P_{n}^{j_{n}}\right)=\delta_{i_{1} j_{1}} \ldots \delta_{i_{n} j_{n}} p\left(i_{1} \ldots i_{n}\right)
$$

When $\mathcal{S}$ is consistent, $p\left(i_{1} \ldots i_{n}\right)$ is the probability of the history $H=\left\{P_{1}^{i_{1}}, \ldots, P_{n}^{i_{n}}\right\}$. We will want later to discuss the properties of individual histories without reference to any fixed consistent set, and we define a consistent history to be a history which belongs to some consistent set $\mathcal{S}$. Finally, we say the set

$$
\mathcal{S}^{\prime}=\left\{\sigma_{1}, \ldots, \sigma_{k}, \tau, \sigma_{k+1}, \ldots, \sigma_{n}\right\}
$$


is a consistent extension of a consistent set of histories $\mathcal{S}=\left\{\sigma_{1}, \ldots, \sigma_{n}\right\}$ by the set of projections $\tau=\left\{Q^{i}: i=1, \ldots, m\right\}$ if $\tau$ is a projective decomposition and $\mathcal{S}^{\prime}$ is consistent.

Suppose now that we have a collection of data defined by the history

$$
H=\left\{P_{1}^{i_{1}}, \ldots, P_{n}^{i_{n}}\right\}
$$

which has non-zero probability and belongs to the consistent set $\mathcal{S}$. This history might, for example, describe the results of a series of experiments or the observations made by an observer. Given a choice of consistent extension $\mathcal{S}^{\prime}$ of $\mathcal{S}$, we can make probabilistic inferences conditioned on the history $H$. For example, if $\mathcal{S}^{\prime}$ has the above form, the histories extending $H$ in $\mathcal{S}^{\prime}$ are $H^{i}=\left\{P_{1}^{i_{1}}, \ldots, P_{k}^{i_{k}}, Q^{i}, P_{k+1}^{i_{k+1}}, \ldots, P_{n}^{i_{n}}\right\}$ and the proposition $Q^{i}$ has conditional probability

$$
p\left(Q^{i} \mid H\right)=p\left(H^{i}\right) / p(H) .
$$

These probabilities and conditional probabilities are the same in every consistent set which includes $H^{i}$ and (hence) $H$. However, when we want to emphasize that the calculation can be carried out in some particular set $\mathcal{S}$, we will attach a suffix. For example, we might write $p_{\mathcal{S}^{\prime}}\left(Q^{i} \mid H\right)=p_{\mathcal{S}^{\prime}}\left(H^{i}\right) / p_{\mathcal{S}}(H)$ for the above equation.

The formalism itself gives no way of choosing any particular consistent extension. In the view of the original developers of the consistent histories approach, the different $\mathcal{S}^{\prime}$ are to be thought of as ways of producing possible pictures of the past and future physics of the system which, though generally incompatible, are all equally valid. More formally, they can be seen as incompatible logical structures which allow different classes of inferences from the data 16, 17]. 
It is this freedom in the choice of consistent extension which, it has been argued elsewhere $9,18,19,20,21,22,23,10$, gives rise to the most serious problems in the consistent histories approach. Standard probabilistic predictions and deterministic retrodictions can be reproduced in the consistent histories formalism by making an ad hoc choice of consistent set, but cannot be derived from the formalism itself. In fact, it is almost never possible to make any unambiguous predictions or retrodictions: there are almost always an infinite number of incompatible consistent extensions of the set containing a given history dataset [9,20].

The problem is not simply that the formalism supplies descriptions of physics which are complementary in the standard sense, although that in itself is sufficient to ensure that the formalism is only very weakly predictive. Even on the assumption that we will continue to observe quasiclassical physics, no known interpretation of the formalism allows us to derive the predictions of classical mechanics and Copenhagen quantum theory. [18]. Hence the set selection problem: probabilistic predictions can only be made conditional on a choice of consistent set, yet the consistent histories formalism gives no way of singling out any particular set or sets as physically interesting.

One possible solution to the set selection problem would be an axiom which identifies a unique physically interesting set, or perhaps a class of such sets, from the initial state and the dynamics. Another would be the identification of a physically natural measure on the space of consistent sets, according to which the physically relevant consistent set is randomly chosen. Possible set selection criteria have been investigated [24,25], but no generally workable criterion has emerged.3

3 With different motivations, Gell-Mann and Hartle have explored a "strong decoherence" 


\section{Consistent Sets and Contrary Inferences: A Brief Review}

A further reason for believing that the consistent histories formalism is at best incomplete comes from considering the logical relations among events in different consistent sets. We say that two projection operators $P$ and $Q$ are complementary if they do not commute: $P Q \neq Q P$. We say that they are contradictory if they sum to the identity, so that $P=1-Q$ and $P Q=Q P=0$, and that they are contrary if they are orthogonal and not contradictory, so that $P Q=Q P=0$ and $P<1-Q$.

Contradictory inferences are never possible in the consistent histories formalism, but it is easy to find examples of contrary inferences from the same data. 19] For instance, consider a quantum system whose hamiltonian is zero and whose Hilbert space $\mathcal{H}$ has dimension greater than two, prepared in the state $|a\rangle$. Suppose that the system is left undisturbed from time 0 until time $t$, when it is observed in the state $|c\rangle$, where $0<$ $|\langle a \mid c\rangle| \leq 1 / 3$ : i.e. a single quantum measurement is made, and the outcome probability is less than $1 / 9$. In consistent histories language, we have initial density matrix $\rho=|a\rangle\langle a|$ and the single datum corresponding to the history $H=\left\{P_{c}\right\}$ from the consistent set $\mathcal{S}=\left\{\left\{P_{c}, 1-P_{c}\right\}\right\}$, where the projection $P_{c}=|c\rangle\langle c|$ is taken at time $t$.

Now consider consistent extensions of $\mathcal{S}$ of the form $\mathcal{S}_{b}=\left\{\left\{P_{b}, 1-P_{b}\right\},\left\{P_{c}, 1-P_{c}\right\}\right\}$ where $P_{b}=|b\rangle\langle b|$ for some normalised vector $|b\rangle$ with the property that

$$
\langle c \mid b\rangle\langle b \mid a\rangle=\langle c \mid a\rangle \text {. }
$$

It is easy to verify that $\mathcal{S}_{b}$ is consistent and that the conditional probability of $P_{b}$ given $H$ is 1 . It is also easy to see that there are at least two mutually orthogonal vectors $|b\rangle$ criterion which is intended to reduce the number of consistent sets. 26] However, under their present definition, every consistent set is strongly decoherent. 10 
satisfying (4.1). For example, let $\left|v_{1}\right\rangle,\left|v_{2}\right\rangle,\left|v_{3}\right\rangle$ be orthonormal vectors and take $|a\rangle=\left|v_{1}\right\rangle$ and $|c\rangle=\lambda\left|v_{1}\right\rangle+\mu\left|v_{2}\right\rangle$, where $|\lambda|^{2}+|\mu|^{2}=1$. Then the vectors

$$
\left|b_{ \pm}\right\rangle=\left(|\lambda|^{2}+\frac{|\mu|^{2}}{x}\right)^{-1 / 2}\left(\lambda\left|v_{1}\right\rangle+\frac{\mu}{x}\left|v_{2}\right\rangle \pm \frac{(x-1)^{1 / 2} \mu}{x}\left|v_{3}\right\rangle\right)
$$

both satisfy (4.1) and are orthogonal if $x$ is real and $x^{2}|\lambda|^{2}=(x-2)\left(1-|\lambda|^{2}\right)$, which has solutions for $|\lambda| \leq 1 / 3$. Thus the consistent sets $\mathcal{S}_{b_{ \pm}}$give contrary probability one retrodictions.

Some brief historical remarks are in order. The existence of contrary inferences in the consistent histories formalism, though easy to show, was noticed only quite recently. In particular, it was not known to the formalism's original developers. 1 It was first explicitly pointed out, and its implications for the consistent histories formalism were first examined, in Ref. [19]. Further discussion can be found in Refs. [27,28]. However, a noteworthy earlier consistent histories analysis of an example in which contrary inferences arise can be found in a critique by Cohen[29] of Aharonov and Vaidman's interpretation[30] of one of their intriguing examples of pre- and post-selection.5 As noted in Ref. [19], Cohen's analysis miscontrues the consistency criterion: however, this error does not affect its derivation of contrary inferences.

Now, the existence of contrary inferences in the consistent histories formalism needs to be interpreted with care. It is not true that, in any given consistent set, two different contrary propositions can be inferred with probability one. The inferences made within any given consistent set lead to no contradiction. The picture of physics given by any

\footnotetext{
4 I am grateful to Bob Griffiths, Jim Hartle, and Roland Omnès for helpful correspondence on this point.

5 I am grateful to Oliver Cohen and Lucien Hardy for drawing this reference to my attention.
} 
given consistent set may or may not be considered natural or plausible - depending on one's intuition and the criteria one uses for naturality — but it is not logically selfcontradictory. It is however true, as a mathematical statement about the properties of the consistent histories formalism, that the propositions inferred in the two different sets correspond to contrary projections. The formalism makes no physical distinction among different consistent sets, and so requires us to conclude that two equally valid pictures of physics can be given, in which contrary events take place.

To put it more formally, the consistent histories approach can be interpreted as setting out rules of reasoning according to which, although physics can be described by any of infinitely many equally valid pictures, only one of those pictures may be considered in any given argument. Such an interpretation ensures - tautologically - that no logical contradiction arises, even when the pictures contain contrary inferences.

The consistent histories formalism, in other words, gives a set of rules for producing possible pictures of physics within quantum theory, and these rules themselves lead to no logical inconsistency. However, consistent historians claim much more, arguing that the formalism defines a natural and scientifically unproblematic interpretation of quantum theory. Indeed, the consistent histories literature tends to suggest that the descriptions of physics given by consistent historians are simply and evidently the correct descriptions which emerge from quantum theory, so that, in querying them, one necessarily queries quantum theory itself 6

This seems patently false. The most basic premise of consistent historians - that

6 For example, the consistent histories interpretation of quantum mechanics has been referred to as "the interpretation of quantum mechanics" [16] and even as simply "quantum mechanics" [31]. 
quantum theory is correctly interpreted by some sort of many-picture scheme - leads to such trouble in explaining which particular picture we see, and why, that cautious scepticism seems only appropriate. Even if the premise were accepted, it would be essential to ask, of any particular many-picture scheme, whether its assumptions are natural and whether the descriptions of nature it produces are physically plausible or scientifically useful. The particular equations used to define consistent sets are, after all, simply interesting guesses: there is no compelling theoretical justification for them, and indeed, several different definitions of consistency have been proposed[[1, 田, 32].

My own view is that there are a number of compelling reasons for regarding the consistent histories interpretation, as it is presently understood, as scientifically unsatisfactory. However, as these questions have been explored in some detail elsewhere $9,18,19,27,28,20-$ 23, 10], I here comment only on two specific problems raised by contrary inferences.

First, the fact that we are to take as equally valid and correct pictures of physics which include contrary inferences goes against many well developed intuitions. No argument based on intuition alone can be conclusive, but I think it must be granted that this one has some force. What use, it may reasonably be asked, is there in saying that in one picture of reality a particle genuinely went, with probability one, through slit $\mathrm{A}$, and that in another picture the particle went, also with probability one, through the disjoint slit B? Why should we take either picture seriously, given the other?

On this point, it is worth noting that one of the advertised merits of the consistent histories formalism[1],16, 17], is that it, unlike the Copenhagen interpretation, accommodates some (arguably) plausible intuitions about the behaviour of microsystems in between ob- 
servations. For example, the formalism allows us to say - albeit only as one of an infinite number of incompatible descriptions - that a particle observed at a particular detector was travelling towards that detector before the observation, and that a particle measured to have spin component $\sigma_{x}=s_{x}$ had that spin before the measurement took place. As Griffiths and Omnès note, [1, 16] informal discussions of experiments are often framed in terms which, if taken literally, suggest that we can make this sort of statement about microsystems before a measurement is carried out. ("Was the beam correctly aligned going into the second interferometer?", or "Do you think something crazy in the electronics might have triggered [detector] number 3 just before the particle got there?" [1], for example.) Their intuition is that a good interpretation of quantum theory ought to give a way of allowing us to take such statements literally — a criterion which, they suggest, the consistent histories approach satisfies.

The intuition is, of course, controversial. A counter-intuition, which most interpretations of quantum theory support, is that any description of a microsystem before a measurement is carried out should be independent of the result of that measurement.

In any case, to the extent that any intuition is offered as a justification of the formalism, it seems reasonable to consider the fact that the formalism violates other strongly held intuitions. Few experimenters, after all, can ever have intuitively concluded that the entire flux of their beam can sensibly be thought of as having followed any of several macroscopically distinct paths through the apparatus. Yet this is what the above example, translated into an interference experiment, implies.

The second, and probably deeper, problem is that it seems very hard to justify the 
distinction, which consistent historians are forced to draw, between contradictory inferences, which are regarded as a priori unacceptable, and contrary inferences, which are regarded as unproblematic. Some justification seems called for, since the distinction is not an accidental feature: it is not that the formalism, for unrelated reasons, simply happens to exclude one type of inference and include the other. The definition of consistency is motivated precisely by the notion that, when two different sets allow a calculation of the probability of the same event (belonging, in the simplest case, to a single history in one set and a combination of two histories in the other), the calculations should agree. This requires in particular that contradictory propositions $P$ and $(1-P)$ can never be inferred, since if the probability of $P$ is one in any set, it must be one in all sets, and so the probability of $(1-P)$ must be zero in all sets.

Now this last requirement is not absolutely essential, sensible though it may seem. No logical contradiction arises in an interpretation of quantum theory which follows the basic interpretational ideas of the consistent histories formalism but which accepts all complete sets of disjoint quantum histories, whether consistent or not, as defining valid pictures of physics [9, 10]. In this "inconsistent histories" interpretation, contradictory inferences can generally be made by using different pictures. This possibility is excluded by a deliberate theoretical choice.

It seems natural, then, having made this choice, to look for ways in which contrary inferences can similarly be excluded. This is the line of thought pursued below. Note, however, that the problem of contrary inferences is not the only motivation for the ideas introduced below. Whatever one's view of the consistent histories formalism, it is inter- 
esting that an alternative formalism can be defined relatively simply. It seems fruitful to ask which, if either, is to be preferred, and why. And, as we will see, ordered consistent sets raise independently interesting questions about the quasiclassical world we actually observe.

\section{Relation of Contrary Inferences and Subspace Implications}

A contrary inference arises when there exist two consistent sets, $\mathcal{S}_{1}$ and $\mathcal{S}_{2}$, both containing a history $H$, with the property that there are orthogonal propositions $P_{1}$ and $P_{2}$ which are implied by $H$ in the respective sets, so that — temporarily adding set suffices for clarity - we have

$$
p_{\mathcal{S}_{1}}\left(P_{1} \mid H\right)=p_{\mathcal{S}_{2}}\left(P_{2} \mid H\right)=1 .
$$

Now $p_{\mathcal{S}_{2}}\left(\left(1-P_{2}\right) \mid H\right)=0$, and since the probabilities are set-independent and $p_{\mathcal{S}_{1}}\left(P_{1} \mid H\right)$ is nonzero, we cannot have $P_{1}=1-P_{2}$. Hence, since $P_{1}$ and $P_{2}$ are orthogonal, we have that $P_{1}<1-P_{2}$. Since $p\left(P_{1} \mid H\right)=1$ and $p\left(\left(1-P_{2}\right) \mid H\right)=0$, this pair of projections violates the subspace implication $P_{1} \Rightarrow 1-P_{2}$. That is, a contrary inference implies the existence of consistent histories $H$ and $H^{\prime}$, belonging to different consistent sets and agreeing on all but one projector, such that $H$ has non-zero probability, $H^{\prime}$ has zero probability, and $H<H^{\prime}$ : in the example of the last section, for instance, we have $H=\left\{P_{b_{+}}, P_{c}\right\}$ and $H^{\prime}=\left\{\left(1-P_{b_{-}}\right), P_{c}\right\}$

Clearly, according to the consistent histories formalism, an observation of the datum $P_{b_{+}}$cannot be taken to imply an observation of the strictly larger projector $\left(1-P_{b_{-}}\right)$. To make that inference would lead directly to a contradiction, in the form of the realisation 
of a probability zero history, if $P_{c}$ were subsequently observed.

Now it is easy to produce real world examples of contrary inferences, so long as those inferences are of unobserved quantities. As we have seen, it requires only a threedimensional quantum system, prepared in one state, isolated, and then observed in another state - hardly a taxing experiment.

It is not obvious, though, that we can produce examples where subspace implications fail in a realistic consistent histories description of observations of laboratory experiments, or more generally of macroscopic quasiclassical physics. That general consistent histories violate subspace implications need not necessarily imply that the particular consistent histories used to recover standard descriptions of real world physics do so. Both in order to address this question, and for its own sake, it is interesting to ask whether there might be any alternative treatment of quantum theory within the consistent histories framework which respects subspace implications, at least when they relate two consistent histories. The next section suggests such a treatment.

\section{Ordered Consistent Sets of Histories}

We have already seen that there is a natural partial ordering for each of the standard representations of quantum histories in the consistent histories approach. The probability weight defines a second partial ordering: $H \prec H^{\prime}$ if $p(H)<p\left(H^{\prime}\right)$. The violation of subspace implications reflects the disagreement between these two partial orderings in the consistent histories formalism: we can have both $H<H^{\prime}$ and $H \succ H^{\prime}$. The aim of this section is to develop a history-based interpretation which restricts attention to collections of quantum histories on which the two orderings do not disagree. 
We begin at the level of individual quantum histories, defining an ordered consistent history, $H$, to be a consistent history with the properties that:

(i) for all consistent histories $H^{\prime}$ with $H^{\prime} \geq H$ we have that $p\left(H^{\prime}\right) \geq p(H)$;

(ii) for all consistent histories $H^{\prime}$ with $H^{\prime} \leq H$ we have that $p\left(H^{\prime}\right) \leq p(H)$.

Recall that a consistent history is any quantum history which belongs to some consistent set of histories. Properties (i) and (ii) hold trivially for histories $H$ and $H^{\prime}$ which belong to the same consistent set: it is the comparison across different sets which makes them useful constraints.

We now define an ordered consistent set of histories to be a complete set of exclusive alternative histories, each of which is ordered consistent. We can then define an ordered consistent histories approach to quantum theory in precise analogy to the consistent histories approach, using the same definition of probability weight and the same interpretation, simply declaring by fiat that only ordered consistent sets of histories are to be considered

The following lemmas show that, within the projection operator formulation, ordered consistent sets of histories do indeed exist.8

Lemma 1: $\quad$ Any consistent history $H=\left\{P_{1}, \ldots, P_{n}\right\}$ defined by a series of projections which include a minimal projection $P_{j}$, so that $P_{i} \geq P_{j}$ for all $\mathrm{i}$, is an ordered consistent history.

7 Note that there are other collections of quantum histories on which the orderings do not disagree. For example, if all the consistent histories $H$ that violate (i) are eliminated, the remainder form a collection on which the orderings do not disagree and which is not obviously identical to the ordered consistent histories, and similarly for (ii). It might be interesting to explore such alternatives, but we restrict attention to the ordered consistent histories here.

8 I am grateful to Bob Griffiths for suggesting a slight extension of Lemma 1. 
Proof: $\quad$ Suppose $H^{\prime}=\left\{P_{1}^{\prime}, \ldots, P_{n}^{\prime}\right\}$ is a larger history than $H$, and write $P_{i}^{\prime}=$ $P_{j}+Q_{i}$. We have that

$$
\begin{aligned}
p\left(H^{\prime}\right) & =\operatorname{Tr}\left(P_{n}^{\prime} \ldots P_{1}^{\prime} \rho P_{1}^{\prime} \ldots P_{n}^{\prime}\right) \\
& =\operatorname{Tr}\left(\left(P_{j}+Q_{n}\right) \ldots\left(P_{j}+Q_{1}\right) \rho\left(P_{j}+Q_{1}\right) \ldots\left(P_{j}+Q_{n}\right)\right) \\
& =\operatorname{Tr}\left(P_{j} \rho\right)+\operatorname{Tr}\left(Q_{n} \ldots Q_{1} \rho Q_{1} \ldots Q_{n}\right) \\
& \geq \operatorname{Tr}\left(P_{j} \rho\right) \\
& =p(H) .
\end{aligned}
$$

Now suppose that $H^{\prime}$ is smaller than $\mathrm{H}$. Then in particular $P_{j}^{\prime} \leq P_{j}$ and we have that

$$
\begin{aligned}
p\left(H^{\prime}\right) & \leq \operatorname{Tr}\left(P_{j}^{\prime} \rho\right) \\
& \leq \operatorname{Tr}\left(P_{j} \rho\right) \\
& =p(H) .
\end{aligned}
$$

Lemma 2: $\quad$ Let $\mathcal{S}=\left\{\sigma_{1}, \ldots, \sigma_{n}\right\}$ be a consistent set of histories defined by a series of projective decompositions, of which one, $\sigma_{j}$, has the property that for each projection $P$ in $\sigma_{j}$, and for every $i$, we have that there is precisely one projection $Q$ in $\sigma_{i}$ with the property that $Q \geq P$ (so that all of the other projections in $\sigma_{i}$ are contrary to $P$ ). Then $\mathcal{S}$ is an ordered consistent set of histories.

Proof: $\quad$ Each of the histories of non-zero probability in $\mathcal{S}$ satisfies the conditions of Lemma 1 and so is ordered consistent. Each of the histories of zero probability in $\mathcal{S}$ is of the form $H=\{\ldots, P, \ldots, Q, \ldots\}$, where $P$ and $Q$ are contrary projections. Now any consistent history smaller than $H$ therefore also contains a pair of contrary projections $P^{\prime} \leq P$ and $Q^{\prime} \leq Q$. By the consistency axioms, its probability is less than or equal to 
$\operatorname{Tr}\left(Q^{\prime} P^{\prime} \rho P^{\prime} Q^{\prime}\right)=0$, and thus must also be zero. Hence $H$ is ordered consistent, since any consistent history larger than $H$ has probability greater than or equal to zero.

\section{Ordered Consistent Sets and Quasiclassicality}

A formalism based on ordered consistent sets of histories obviously defines a more strongly predictive version of quantum theory than that defined by the existing consistent histories framework, since it allows strictly fewer sets as possible descriptions of physics. But can it describe our empirical observations?

The question subdivides. Are quasiclassical domains generally ordered consistent sets of histories? Is our own quasiclassical domain one? If not, are its histories generally ordered when compared to histories belonging to other consistent sets defined by projections onto ranges of the same quasiclassical variables? For example, can we show that consistent histories defined by projections onto ranges of densities for chemical species in small volumes are generally ordered with respect to one another? If so, then the type of subspace implication which is generally used in analyses of observations could still be justified. Finally, if either of the previous two properties fail to hold, it would be useful to quantify the extent to which they fail.

Answering any of these questions definitively may require — and, it might be hoped, help to develop - a deeper understanding of quasiclassicality than is available to us at

present. I at any rate do not know the answers, and can only offer the questions as interesting ones whose resolution would have significant implications. At least ordered consistency does not seem to fall at the first hurdle: ordered consistent sets are shown below to be adequate to describe quasiclassicality in simple models. 
As a simple example, consider the following model of a series of successive measurements of the spin of a spin- $1 / 2$ particle about various axes. We use a vector notation for the particle states, so that if $\mathbf{u}$ is a unit vector in $R^{3}$ the eigenstates of $\sigma$.u are represented by $| \pm \mathbf{u}\rangle$. With the analogy of a pointer state in mind, we use the basis $\left\{|\uparrow\rangle_{k},|\downarrow\rangle_{k}\right\}$ to represent the $k^{\text {th }}$ environment particle state, together with the linear combinations $| \pm\rangle_{k}=\left(|\uparrow\rangle_{k} \pm|\downarrow\rangle_{k}\right) / \sqrt{2}$. We compactify the notation by writing environment states as single kets, so that for example $|\uparrow\rangle_{1} \otimes \cdots \otimes|\uparrow\rangle_{n}$ is written as $|\uparrow \ldots \uparrow\rangle$, and we take the initial state $|\psi(0)\rangle$ to be $|\mathbf{v}\rangle \otimes|\uparrow \ldots \uparrow\rangle$.

The interaction between the system and the $k^{\text {th }}$ environment particle is chosen so that it corresponds to a measurement of the system spin along the $\mathbf{u}_{k}$ direction, so that the states evolve as follows:

$$
\begin{gathered}
\left|\mathbf{u}_{k}\right\rangle \otimes|\uparrow\rangle_{k} \rightarrow\left|\mathbf{u}_{k}\right\rangle \otimes|\uparrow\rangle_{k}, \\
\left|-\mathbf{u}_{k}\right\rangle \otimes|\uparrow\rangle_{k} \rightarrow\left|-\mathbf{u}_{k}\right\rangle \otimes|\downarrow\rangle_{k} .
\end{gathered}
$$

A simple unitary operator that generates this evolution is

$$
U_{k}(t)=P\left(\mathbf{u}_{k}\right) \otimes I_{k}+P\left(-\mathbf{u}_{k}\right) \otimes \exp \left(-i \theta_{k}(t) F_{k}\right)
$$

where $P(\mathbf{x})=|\mathbf{x}\rangle\langle\mathbf{x}|$ and $F_{k}=i|\downarrow\rangle_{k}\left\langle\left.\uparrow\right|_{k}-i \mid \uparrow\right\rangle_{k}\left\langle\downarrow||_{k}\right.$. Here $\theta_{k}(t)$ is a function defined for each particle $k$, which varies from 0 to $\pi / 2$ and represents how far the interaction has progressed. We define $P_{k}( \pm)=| \pm\rangle_{k}\left\langle \pm\left.\right|_{k}\right.$, so that $F_{k}=P_{k}(+)-P_{k}(-)$.

The Hamiltonian for this interaction is thus

$$
H_{k}(t)=i \dot{U}_{k}(t) U_{k}^{\dagger}(t)=\dot{\theta}_{k}(t) P\left(-\mathbf{u}_{k}\right) \otimes F_{k},
$$

in both the Schrödinger and Heisenberg pictures. We write the extension of $U_{k}$ to the total Hilbert space as

$$
V_{k}=P\left(\mathbf{u}_{k}\right) \otimes I_{1} \otimes \cdots \otimes I_{n}+P\left(-\mathbf{u}_{k}\right) \otimes I_{1} \otimes \cdots \otimes I_{k-1} \otimes \exp \left(-i \theta_{k}(t) F_{k}\right) \otimes I_{k+1} \otimes \cdots \otimes I_{n}
$$


We take the system particle to interact initially with particle 1 and then with consecutively numbered ones, and there is no interaction between environment particles, so that the evolution operator for the complete system is

$$
U(t)=V_{n}(t) \ldots V_{1}(t)
$$

with each factor affecting only the Hilbert spaces of the system and one of the environment spins.

We suppose, finally, that the interactions take place in disjoint time intervals and that the first interaction begins at $t=0$, so that the total Hamiltonian is simply

$$
H(t)=\sum_{k=1}^{n} H_{k}(t),
$$

and we have that $\theta_{1}(t)>0$ for $t>0$ and that, if $0<\theta_{k}(t)<\pi / 2$, then $\theta_{i}(t)=$ $\pi / 2$ for all $i<k$ and $\theta_{i}(t)=0$ for all $i>k$.

This model has been used elsewhere [25,33] in order to explore algorithms which might select a single physically natural consistent set when the physics is determined by the simplest type of system-environment interaction. It is particularly well suited to such an analysis, since the dynamics are chosen so as to allow a simple and quite elegant classification 33] of all the consistent sets built from projections onto subspaces defined by the Schmidt decomposition. Apart from this, though, the model is unexceptional - one of the simpler variants among the many models used in the literature to investigate the decoherence of system states by measurement-type interactions with an environment.

To give a physical interpretation of the model, we take it that the environment "pointer" variables assume definite values after their respective interactions with the system. That is, after the $k^{\text {th }}$ interaction, the $k^{\text {th }}$ environment particle is in one of the states 
$|\uparrow\rangle_{k}$ and $|\downarrow\rangle_{k}$ : the probabilities of each of these outcomes depend on the outcome of the previous measurement (or, in the case of the first measurement, on the initial state) via the standard quantum mechanical expressions.

This description can be recovered from the consistent histories formalism by choosing the consistent set $\mathcal{S}_{1}$, defined by the decompositions

$$
\begin{aligned}
& \left\{I \otimes\left|\epsilon_{1}\right\rangle_{1}\left\langle\left.\epsilon_{1}\right|_{1} \otimes I \otimes \cdots \otimes I: \epsilon_{1}=\uparrow \text { or } \downarrow\right\} \text { at time } t_{1},\right. \\
& \left\{I \otimes\left|\epsilon_{1}\right\rangle_{1}\left\langle\left.\epsilon_{1}\right|_{1} \otimes \mid \epsilon_{2}\right\rangle_{2}\left\langle\left.\epsilon_{2}\right|_{2} \otimes \cdots \otimes I: \epsilon_{1}, \epsilon_{2}=\uparrow \text { or } \downarrow\right\} \text { at time } t_{2},\right. \\
& \quad \cdots \\
& \left\{I \otimes\left|\epsilon_{1}\right\rangle_{1}\left\langle\left.\epsilon_{1}\right|_{1} \otimes \mid \epsilon_{2}\right\rangle_{2}\left\langle\left.\epsilon_{2}\right|_{2} \otimes \cdots \otimes \mid \epsilon_{n}\right\rangle_{n}\left\langle\left.\epsilon_{n}\right|_{n}: \epsilon_{1}, \epsilon_{2}, \ldots, \epsilon_{n}=\uparrow \text { or } \downarrow\right\} \text { at time } t_{n} .\right.
\end{aligned}
$$

Clearly, the histories of non-zero probability in $\mathcal{S}_{1}$ take the form

$$
\begin{aligned}
H_{\epsilon_{1}, \ldots, \epsilon_{n}}=\{ & I \otimes I \otimes I \otimes \cdots \otimes I, \\
& I \otimes\left|\epsilon_{1}\right\rangle_{1}\left\langle\left.\epsilon_{1}\right|_{1} \otimes I \otimes \cdots \otimes I,\right. \\
& I \otimes\left|\epsilon_{1}\right\rangle_{1}\left\langle\left.\epsilon_{1}\right|_{1} \otimes \mid \epsilon_{2}\right\rangle_{2}\left\langle\left.\epsilon_{2}\right|_{2} \otimes I \otimes \cdots \otimes I,\right. \\
& \ldots, \\
& I \otimes\left|\epsilon_{1}\right\rangle_{1}\left\langle\left.\epsilon_{1}\right|_{1} \otimes \mid \epsilon_{2}\right\rangle_{2}\left\langle\left.\epsilon_{2}\right|_{2} \otimes \cdots \otimes \mid \epsilon_{n}\right\rangle_{n}\left\langle\left.\epsilon_{n}\right|_{n}\right\},
\end{aligned}
$$

for sequences $\left\{\epsilon_{1}, \ldots, \epsilon_{n}\right\}$, each element of which takes the value $\uparrow$ or $\downarrow$. Their probabilities, defined by the decoherence functional, are precisely those which would be obtained from standard quantum theory by treating each interaction as a measurement:

$$
p\left(H_{\epsilon_{1}, \ldots, \epsilon_{n}}\right)=\left(\frac{1+a_{1} \mathbf{v} \cdot \mathbf{u}_{\mathbf{1}}}{2}\right)\left(\frac{1+a_{2} \mathbf{u}_{\mathbf{1}} \cdot \mathbf{u}_{\mathbf{2}}}{2}\right) \ldots\left(\frac{1+a_{n} \mathbf{u}_{\mathbf{n}-\mathbf{1}} \cdot \mathbf{u}_{\mathbf{n}}}{2}\right),
$$

where, letting $\epsilon_{0}=\uparrow$, we define $a_{i}=1$ if $\epsilon_{i}$ and $\epsilon_{i-1}$ take the same value, and $a_{i}=-1$ otherwise. 
Now $\mathcal{S}_{1}$ is defined by a nested sequence of increasingly refined projective decompositions, all of whose projections commute - a relation which is unaltered by moving to the Heisenberg picture. It therefore satisfies the conditions of Lemma 2 above, and so is ordered consistent.

This argument clearly generalizes: in any situation in which Hilbert space factorizes into system and environment degrees of freedom, where the self-interactions of the latter are negligible, any consistent set defined by nested commuting projections onto the environment variables is ordered consistent. The model considered above is a particularly crude example: more sophisticated, and phenomenologically somewhat more plausible, examples of this type are analysed in, for example, Refs. [26, 32].

No sweeping conclusion can be drawn from this, since it is generally agreed that familiar quasiclassical physics is not well described in general — at least in any obvious way - by models of this type. (Again, a detailed discussion of the limitations of such models can be found in Refs. [26, 32].) In other words, while it would be hard to defend the hypothesis that familiar quasiclassical sets are generally ordered consistent if sets of the type $\mathcal{S}_{1}$ were not, the fact that they are is certainly not sufficient evidence. It would be good to find sharper tests of the hypothesis, perhaps for example by developing further the phenomenological investigations of quasiclassicality pursued in Ref. [26]. Meanwhile, the questions raised earlier in this section remain unresolved.

On the other hand, it would be difficult to make a watertight case that ordered consistent sets are definitely inadequate to describe real-world physics, for the following reason. First, it seems hard to exclude the possibility that the initial state is pure, so let us 
temporarily suppose that it is: $\rho=|\psi\rangle\langle\psi|$. As Gell-Mann and Hartle point out 32], we can then associate to every consistent set of histories, $\mathcal{S}$, a nested set of commuting projections defining what they term generalized records. The consistent set defines a resolution of the initial state into history vectors,

$$
|\psi\rangle=\sum_{i_{1}, \ldots, i_{n}} P_{n}^{i_{n}} \ldots P_{1}^{i_{1}}|\psi\rangle,
$$

which are guaranteed to be orthogonal by the consistency condition (3.2). We can thus find at least one set of orthogonal projection operators $\left\{R_{I}\right\}$, indexed by sets of the form $I=\left\{i_{1} \ldots i_{n}\right\}$, which project onto the history vectors and sum to the identity:

$$
\begin{aligned}
R_{i_{1} \ldots i_{n}}|\psi\rangle & =P_{n}^{i_{n}} \ldots P_{1}^{i_{1}}|\psi\rangle, \\
\sum_{I} R_{I} & =I, \\
R_{I} R_{J} & =\delta_{I J} R_{J} .
\end{aligned}
$$

We can thus 32] construct a set $\mathcal{S}^{\prime}$, with the same history vectors and the same probabilities as $\mathcal{S}$, built from a nested sequence of commuting projections defined by sums of the $R_{I}$. And, as we have seen, sets of this type are ordered consistent.

There is no reason to expect the projections defining the set $\mathcal{S}^{\prime}$ to be closely related to those defining $\mathcal{S}$. In particular, the fact that $\mathcal{S}$ is a quasiclassical domain certainly does not imply that $\mathcal{S}^{\prime}$ is likely to be: its projections are not generally likely to be interpretable in terms of familiar variables. But, as we have already noted, we have no theoretical criterion which identifies a particular consistent set, or a particular type of variable, as fundamentally correct for representing the events we observe. The set $\mathcal{S}^{\prime}$ correctly identifies the history vectors and predicts their probabilities, and we thus could not say for certain (given that we presently have no theory of set selection) that its description of physics is fundamentally incorrect, while that given by $\mathcal{S}$ is fundamentally correct. 
To make this observation is merely to point out a logical possibility. In fact, it would be extremely puzzling if the more complicated and apparently derivative set $\mathcal{S}^{\prime}$ were in some sense more fundamental than the associated quasiclassical domain $\mathcal{S}$. And even if this were somehow understood to be true in principle, we would still need to understand the relationship between between ordered consistency and quasiclassicality in order to say whether or not standardly used subspace inferences are in fact justifiable.

\section{Ordering and Ordering Violations: Interpretation}

However, one of the main points of this paper — and the main reason for taking a particular interest in the properties of ordered consistent sets - is that either answer leads to an interesting conclusion.

If our empirical observations can be accounted for by the predictions of an ordered consistent set, then the ordered consistent sets formalism supersedes the current versions of the consistent histories formalism as a predictive theory. Alternatively, if the predictions of ordered consistent histories quantum theory are false, then either the consistent histories framework uses entirely the wrong language to describe histories of events in quantum theory, or we cannot generally rely on subspace implications in analysing our observations.

Either of these last two possibilities would have far-reaching implications for our understanding of nature. It is true that other ways of representing quantum histories are known than those used in the consistent histories formalism, but they arise either in nonstandard versions of quantum theory, such as de Broglie-Bohm theory, or in alternative theories. It is also true that there is no way of logically excluding the possibility that subspace implications generally fail to hold. Any clear violation would, however, lead to 
radical changes in our representation of the world, and in particular to our understanding of the relation between theory and empirical observation.

I would suggest, however, that any version of closed system quantum theory in which the two orderings disagree leads to radical new interpretational problems. The fundamental problem is that, supposing that the world we experience is described by one particular realised quantum history, we never know - no matter how precise we try to make our observations - exactly what form that history takes. This is not only because we can never completely eliminate imprecision from our experimental observations. A deeper problem is that we have no theoretical understanding of how, precisely, an observation should be represented within quantum theory. We do not know precisely when and where any given observation takes place. Nor do we know whether is fundamentally correct to represent quantum events by projection operators, by quantum effects, by statements associated to space-time regions in path integral quantum theory, or in some other way - let alone precisely which operator, effect, or statement correctly represents any given event. As a result, we are always forced into guesswork and approximation. We are forced to assume, at least as a working hypothesis, that we can find sensible bounds on our observations. Roughly speaking, we assume that we can say, at least, that a photon hit our photographic plate within a certain region, that the observed flux from a distant star was in a certain range, and so forth.9 We assume also that the probability of the actual observations whose precise form we do not know — is bounded by the probability of the observations

\footnotetext{
9 In fact such statements are generally made within statistical confidence limits. To consider statistical statements would complicate the discussion a little, but does not alter the underlying point.
} 
as we approximately represent them. These assumptions ultimately rely on the agreement of two orderings just mentioned: when those orderings disagree, we therefore run into new problems.

It is easy to see, in particular, that this sort of problem arises in any careful consistent histories treatment of quantum cosmology. Suppose, for example, that we have a sequence of cosmological events which we wish to represent theoretically, in order to calculate their probability, given some theory of the boundary conditions. Assuming that the basic principles of the consistent histories formalism are correct, we know that these events should be represented by some history $H$ belonging to some consistent set $\mathcal{S}$. We do not, however, know the precise form of $H$ or of $\mathcal{S}$ : the events are given to us as empirical observations rather than as mathematical constructs.

The best we can then do, following the general principles of the consistent histories formalism, is choose some plausible consistent set $\mathcal{S}^{\prime}$ containing histories $H_{\min }$ and $H_{\max }$ which we guess to have the property $H_{\min }<H<H_{\max }$ : in particular, thus, we choose $H_{\min }<H_{\max }$. Since $H_{\min }$ and $H_{\max }$ belong to the same set, we have that $p\left(H_{\min }\right)<$ $p\left(H_{\max }\right)$. It might naively be hoped that we can derive that $p\left(H_{\min }\right)<p(H)<p\left(H_{\max }\right)$, but since $H$ in general will belong to a different consistent set from $H_{\min }$ and $H_{\max }$, this does not generally follow. There is no way to bound $p(H)$, except (in principle) by performing the enormous task of explicitly calculating the probabilities of all consistent histories bounded by $H_{\min }$ and $H_{\max }$, and there is no way to justify the type of subspace implication — relating observations and true data - that we generally take for granted.

This is not to say that the disagreement of the two orderings necessarily leads to 
logical contradiction. Versions of quantum theory in which the orderings disagree need not be inconsistent, or even impossible to test precisely. They do, though, generally seem to require us to identify precisely the correct representation of our observations in quantum theory. This is generally a far from trivial problem: how are we to tell, a priori, exactly which projection operators represent the results of a series of quantum measurements? It is not impossible to imagine that theoretical criteria could be found which solve the problem, but we certainly do not have such criteria at present.

\section{Conclusions}

Though the criterion of ordering seems mathematically natural, both in the consistent histories approach to quantum theory and in other possible treatments of quantum histories, it raises very unconventional questions. It seems, though, that these questions cannot be avoided in any precise formulation of the quantum theory of a closed system which involves a standard representation of quantum events and which gives a historical account.

There seem to be three possibilities, each of which is interesting. The first is that the representations of quantum histories discussed here, though standard, are not those chosen by nature. Clearly this is a possibility: there are, for example, well known non-standard versions of quantum theory [6], and related theories[7], in which histories are defined by trajectories or other auxiliary variables, and in which subspace implications follow just as in classical physics.

The second possibility is that our quasiclassical domain can be shown to be an ordered 
consistent set. If so, then the ordered consistent histories approach is both predictively stronger than the standard consistent histories approach — since there are fewer ordered consistent sets - and compatible with empirical observation, and hence superior as a predictive theory. If it is compatible with our observations, the ordered consistent histories approach would seem at least as natural as the consistent histories approach.

Even if so, I would not suggest that the ordered consistent histories formalism is the "right" interpretation of quantum theory, and the consistent histories approach the "wrong" one. The ordered consistent histories approach seems almost certain to suffer from many of the same defects as the consistent histories approach, since there are still far too many ordered consistent sets. The aim here is thus not to propose the ordered consistent histories approach as a plausible fundamental interpretation of quantum theory, but to suggest that the range of natural and useful mathematical definitions of types of quantum history is wider than previously understood. This range includes, at least, Goldstein and Page's criterion of linear positivity [34, the various consistency criteria [1, 26, 32, in the literature, and the criterion of ordered consistency introduced here. It seems to me hard to justify taking any of these criteria as defining the fundamentally correct interpretation of quantum theory. On the one hand, physically interesting quantum histories might possibly satisfy any one, or none, of them; on the other hand, most quantum histories satisfying any given criterion seem unlikely to be physically interesting — and precisely which criteria are useful in which circumstances largely remains to be understood.

The third possibility is that our quasiclassical domain is not an ordered consistent set. This would have intriguing theoretical implications. We would have, at least in principle, 
to abandon subspace inferences, and we would ultimately need to understand precisely how to characterise the quantum events which constitute the history we observe. This would raise profound and not easily answerable questions about how we can tell what, precisely, are our empirical observations. It might also, depending on the way in which ordered consistency was violated, and the extent of any violation, raise significant practical problems in the analysis of those observations.

No compelling argument in favour of any one of these possibilities has been given here: it has been shown only that, if quasiclassical sets generally fail to be ordered consistent, they do so in a way too subtle to be displayed in the simplest models.

Another caveat is that the above discussion applied the criterion of ordered consistency only to the simplest representation of quantum histories, in which individual events are represented by projections at a single time. Other representations need to be considered case by case, and our conclusions might not necessarily generalize. For example, the fact that a consistent history built from single time projections is ordered when compared to consistent histories of the same type does not necessarily imply that it is ordered when compared to consistent histories defined by composite events.

Still, the criterion of ordered consistency defines a new version of the consistent histories formulation of quantum theory, which avoids the problems caused by contrary inferences. Its other properties and implications largely remain to be understood. 


\section{Acknowledgements}

I am grateful to Jeremy Butterfield for a critical reading of the manuscript and many thoughtful comments, to Chris Isham and Noah Linden for very helpful discussions of their related work, and to Fay Dowker, Arthur Fine and Bob Griffiths for helpful comments.

I would particularly like to thank Francesco Petruccione for organising the small and

lively meeting at which this work, inter alia, was discussed, and for his patient editorial encouragement.

This work was supported by a Royal Society University Research Fellowship. 


\section{References}

[1] R.B. Griffiths, J. Stat. Phys. 36 (1984) 219.

[2] R.B. Griffiths, Found. Phys. 23 (1993) 1601.

[3] R. Omnès, J. Stat. Phys. 53 (1988) 893.

[4] M. Gell-Mann and J.B. Hartle in Complexity, Entropy, and the Physics of Information, SFI Studies in the Sciences of Complexity, Vol. VIII, W. Zurek (ed.), Addison Wesley, Reading (1990).

[5] C.J. Isham, J. Math. Phys. 23 (1994) 2157.

[6] D. Bohm, Phys. Rev. 85 (1952) 166.

[7] G. Ghirardi, A. Rimini and T. Weber, Phys. Rev. D34 (1986) 470.

[8] R.D. Sorkin, Mod. Phys. Lett. A9 (1994) 3119.

[9] F. Dowker and A. Kent, J. Stat. Phys. 82 (1996) 1575.

[10] A. Kent, in Modern Studies of Basic Quantum Concepts and Phenomena, Proceedings of the 104th Nobel Symposium, Gimo, June 1997, Physica Scripta T76 78-84 (1998).

[11] O. Rudolph, Int. J. Theor. Phys. 35 (1996) 1581.

[12] O. Rudolph, J. Math. Phys. 37 (1996) 5368.

[13] J.B. Hartle, in Quantum Cosmology and Baby Universes, Proceedings of the 1989 Jerusalem Winter School on Theoretical Physics, ed. by S. Coleman, J. Hartle, T. Piran, and S. Weinberg, World Scientific, Singapore (1991).

[14] C.J. Isham and N. Linden, J. Math. Phys. 35 (1994) 5452.

[15] C.J. Isham, N. Linden and S. Schreckenberg, J. Math. Phys. 35 (1994) 6360.

[16] R. Omnès, The Interpretation of Quantum Mechanics, Princeton University Press, Princeton (1994).

[17] R.B. Griffiths, Phys. Rev. A54 (1996) 2759.

[18] A. Kent, Phys. Rev. A54 (1996) 4670.

[19] A. Kent, Phys. Rev. Lett. 78 (1997) 2874.

[20] F. Dowker and A. Kent, Phys. Rev. Lett. 75 (1995) 3038.

[21] J. Paz and W. Zurek, Phys. Rev. D48 (1993) 2728.

[22] I. Giardina and A. Rimini, Found. Phys. 26 (1996) 973.

[23] A. Kent, in Bohmian Mechanics and Quantum Theory: An Appraisal, edited by J. Cushing, A. Fine, and S. Goldstein (Kluwer Academic Press, Dordrecht, 1996).

[24] R. Omnès, Phys. Lett. A 187 (1994) 26.

[25] A. Kent and J. McElwaine, Phys. Rev. A55 (1997) 1703.

[26] M. Gell-Mann and J.B. Hartle, University of California, Santa Barbara preprint UCSBTH-95-28; gr-qc/9509054.

[27] R. Griffiths and J. Hartle, Phys. Rev. Lett. 81 (1998) 1981.

[28] A. Kent, Phys. Rev. Lett. 81 (1998) 1982.

[29] O. Cohen, Phys. Rev. A51 (1995) 4373. 
[30] Y. Aharonov and L. Vaidman, J. Phys. A 24 (1991) 2315.

[31] M. Gell-Mann and J.B. Hartle, University of California, Santa Barbara preprint UCSBTH-94-09; gr-qc/9404013.

[32] M. Gell-Mann and J.B. Hartle, Phys. Rev. D47 (1993) 3345.

[33] J. McElwaine, Phys. Rev. A56 (1997) 1756.

[34] S. Goldstein and D. Page, Phys. Rev. Lett. 74 (1995) 3715. 


\section{Appendix: Ordering and Decoherence Functionals}

This Appendix describes a noteworthy earlier discussion of quantum history orderings, given by Isham and Linden in Sec. IV of Ref. [14, and its relation to the ideas discussed here.

Isham and Linden abstract the basic ideas of the consistent histories formalism in the following way. First, the space $\mathcal{U P}$ of history propositions is taken to be a mathematical structure - an orthoalgebra - with a series of operations and relations obeying certain axioms. In particular, they propose that a partial ordering $\leq$ and an orthogonality relation $\perp$ should be defined on $\mathcal{U P}$ and should obey natural rules, and that $\mathcal{U P}$ should include an identity history 1.

They then introduce a space $\mathcal{D}$ of decoherence functionals, defined to be maps from $\mathcal{U P} \times \mathcal{U P}$ to the complex numbers satisfying certain axioms, and go on to consider whether the axioms defining decoherence functionals should include axioms relating to the ordering in $\mathcal{U P}$.

In the language of standard quantum mechanics, $\mathcal{U P}$ corresponds to the space of all the quantum histories (not only the consistent histories) for a given system, whose Hilbert space and hamiltonian are fixed. Any of several representations of quantum histories could be considered: the relevant part of Isham and Linden's discussion uses the simplest representation of quantum histories, as sequences of projection operators.

The standard quantum mechanical decoherence functional (as appears on the left hand side of (3.2) ) is a member of the space $\mathcal{D}$ in the minimal axiom system Isham and Linden eventually choose. As they remark, though, it would not be a member of $\mathcal{D}$ if the extra ordering axioms they discuss were imposed. Isham and Linden nonetheless consider imposing these ordering axioms, since their aim in the relevant discussion is to investigate generalised algebraic and logical schemes rather than to propose a formalism applicable to standard quantum theory. (They suggest, at the end of section IV, that standard quantum theory might perhaps emerge from some such generalised scheme in an appropriate limit.)

Isham and Linden were, as far as I am aware, the first to investigate possible uses of orderings in developing the consistent histories formalism. It is worth stressing, though, to avoid any possible confusion, that their suggestions pursue the exploration of orderings in a direction orthogonal to the one considered in the present paper. In this paper we restrict attention to standard quantum theory, and propose an alternative histories formalism within that theory, using the standard quantum theoretic decoherence functional throughout. We note also that the subspace implications which underlie our basic scientific worldviews depend for their justification on the assumption that the quasiclassical set describing the physics we observe is an ordered consistent set. Isham and Linden's proposed ordering axioms, on the other hand, exclude standard quantum theory and the standard decoherence functional: they are possible postulates which might be imposed 
on non-standard generalised decoherence functionals in non-standard generalisations of quantum theory.

Isham and Linden give a minimal set of postulated properties for generalised decoherence functionals:

$$
\begin{aligned}
& d(0, \alpha)=0 \text { for all } \alpha \in \mathcal{U P} \\
& d(\alpha, \beta)=d(\beta, \alpha)^{*} \text { for all } \alpha, \beta \in \mathcal{U P} ; \\
& d(\alpha, \alpha) \geq 0 \text { for all } \alpha \in \mathcal{U P} ; \\
& \text { if } \alpha \perp \beta \text { then, for all } \gamma, d(\alpha \oplus \beta, \gamma)=d(\alpha, \gamma)+d(\beta, \gamma) \\
& d(1,1)=1 .
\end{aligned}
$$

They then consider imposing new postulates on decoherence functionals. The first of these - their posited inequality 1 - is that:

$$
\text { for all } d \in \mathrm{D} \text { and for all } \alpha, \beta \text { with } \alpha \leq \beta \text { we have } d(\alpha, \alpha) \leq d(\beta, \beta) \text {. }
$$

As Isham and Linden go on to point out, there are familiar examples in standard quantum theory in which (9.2) is violated for a pair of histories $\alpha \leq \beta$ in which one of the histories (in their case $\alpha$ ) is inconsistent 10

Two further postulates on generalised decoherence functionals are also posited:

$$
\alpha \perp \beta \text { implies } d(\alpha, \alpha)+d(\beta, \beta) \leq 1 \text { for all } d \in \mathrm{D} ;
$$

and

$$
\text { for all } d \in \mathrm{D} \text { and all } \gamma \in \mathcal{U P} \text { we have } d(\gamma, \gamma) \leq 1 \text {. }
$$

Isham and Linden give examples to show that, in standard quantum theory, with the standard decoherence functional, inconsistent histories do not necessarily respect these inequalities either.

Again, the difference from the examples considered in the present paper is worth emphasizing. All of the examples Isham and Linden consider involve inconsistent histories these are all they require in order to investigate possible properties of decoherence functionals applied to arbitrary, not necessarily consistent, quantum histories. These examples are not problematic for the consistent histories approach to quantum theory, according to which the inconsistent histories have no physical significance, and they do not give rise to

10 The suggestion that inequality 1 is true when applied to sequences of projectors onto subsets of configuration space in a path-integral quantum theory is thus misleading: it is easy to find configuration space analogues of these examples. I am grateful to Chris Isham and Noah Linden for discussions of this point. 
new interpretational questions in any conventional quantum histories approach, for essentially the same reason. The discussion in the present paper, on the other hand, looks at the properties of consistent histories in standard quantum theory: we have argued that their failure to respect ordering relations is problematic and explained that it does raise new questions.

Suppose now that we set aside Isham and Linden's motivations, and alter their ordering postulates so that they apply, not to generalised decoherence functionals applied to all quantum histories in an abstract generalisation of quantum theory, but to the standard decoherence functional applied to ordered consistent histories in standard quantum theory. We then obtain the following:

$$
\begin{aligned}
& \text { for all } \alpha, \beta \text { with } \alpha \leq \beta \text { we have } d(\alpha, \alpha) \leq d(\beta, \beta) ; \\
& \qquad \alpha \perp \beta \text { implies } d(\alpha, \alpha)+d(\beta, \beta) \leq 1 ;
\end{aligned}
$$

and

$$
\text { for all } \gamma \text { we have } d(\gamma, \gamma) \leq 1
$$

Here $d$ is the standard decoherence functional, $\alpha, \beta$ and $\gamma$ are now taken to be ordered consistent histories, and $\alpha \perp \beta$ means that $\alpha$ and $\beta$ are disjoint - i.e., there is at least one time at which their respective events are represented by contrary projections.

The first of these equations holds by the definition of an ordered consistent history, but it might perhaps be hoped that the others could restrict the class of histories further. However, the second equation also holds for all ordered consistent histories. To see this, note that $\alpha \perp \beta$ implies that $\beta \leq(1-\alpha)$, and that if $\alpha$ is a consistent history then $(1-\alpha)$ is too. The fact that $\beta$ is ordered consistent thus implies that

$$
p(\beta) \leq p(1-\alpha)=1-p(\alpha)
$$

The third equation, moreover, holds for all consistent histories, ordered or otherwise. It seems, then, that ordered consistency may be the strongest natural criterion that can be defined using the basic ingredients of consistency and ordering. 